\title{
CoREAS simulations of inclined air showers predict refractive displacement of the radio-emission footprint
}

\author{
Marvin Gottowik, ${ }^{a, *}$ Felix Schlüter, ${ }^{b, c}$ Tim Huege $^{b, d}$ and Julian Rautenberg ${ }^{a}$ \\ ${ }^{a}$ Bergische Universität Wuppertal, Wuppertal, Germany \\ ${ }^{b}$ Karlsruher Institut für Technologie, Institut für Astroteilchenphysik, Karlsruhe, Germany \\ ${ }^{c}$ Universidad Nacional de San Martín, Instituto de Tecnologías en Detección y Astropartículas, Buenos \\ Aires, Argentina \\ ${ }^{d}$ Vrije Universiteit Brussel, Astrophysical Institute, Brussels, Belgium \\ E-mail: gottowik@uni-wuppertal.de, felix.schlueter@kit.edu
}

\begin{abstract}
Simulating the radio emission of inclined extensive air showers for a ground-based radio-antenna array we find a systematic displacement of the radio emission with respect to the Monte-Carlo shower impact point. We correct the radio-emission footprint for the asymmetries due to the superposition of geomagnetic and charge-excess radiation as well as for the early-late effect. The remaining displacement is found to be $\sim 1500 \mathrm{~m}$ along the ground plane for showers with a zenith angle of $85^{\circ}$, which is relevant for air shower detectors. A model describing this displacement by refraction in the atmosphere based on Snell's law yields good agreement with our observations from CoREAS simulations. We thus conclude that the displacement is caused by refraction in the atmosphere.
\end{abstract}

$37^{\text {th }}$ International Cosmic Ray Conference (ICRC 2021)

July 12th - 23rd, 2021

Online - Berlin, Germany

\footnotetext{
${ }^{*}$ Presenter
} 


\section{Introduction}

The lateral distribution of the radio signal of an extensive air shower is affected by a strong asymmetry arising from the superposition of the geomagnetic and charge-excess emission caused by their individual polarization patterns [1]. An additional early-late asymmetry becomes relevant for inclined air showers with a zenith angle larger than $60^{\circ}$ [2]. Here, we investigate a third effect in the radio-emission footprint of inclined air showers simulated with CoREAS [3], which we have originally published in [4].

The radio-emission footprint is found to be displaced from the Monte-Carlo (MC) shower axis due to refraction of the radio emission in the atmosphere as shown in Fig. 1. We have simulated a highly inclined air shower with a zenith angle of $85^{\circ}$ arriving from the South for four different atmospheric refractivity profiles. The lateral distribution of the radio emission in terms of the energy fluence $f$ is shown along the positive and negative $\mathbf{v} \times(\mathbf{v} \times \mathbf{B})$ axis ( $\mathbf{v}$ : shower axis, $\mathbf{B}$ : magnetic field, the positive $\mathbf{v} \times(\mathbf{v} \times \mathbf{B})$ axis points in the incoming direction of the shower). As expected, the energy fluence is symmetric on both axes for a constant value of the refractive index $n$ throughout the atmosphere ( 1.00003 is approximately the value of $n$ at the height of $X_{\max }$ ). However, an apparent asymmetry is observed for a changing refractive index following the density gradient in the atmosphere which is enhanced when doubling the refractivity $n-1$ throughout the atmosphere. This effect can be understood as a refractive displacement of the radio-emission footprint, correcting for the displacement we find this asymmetry to vanish.

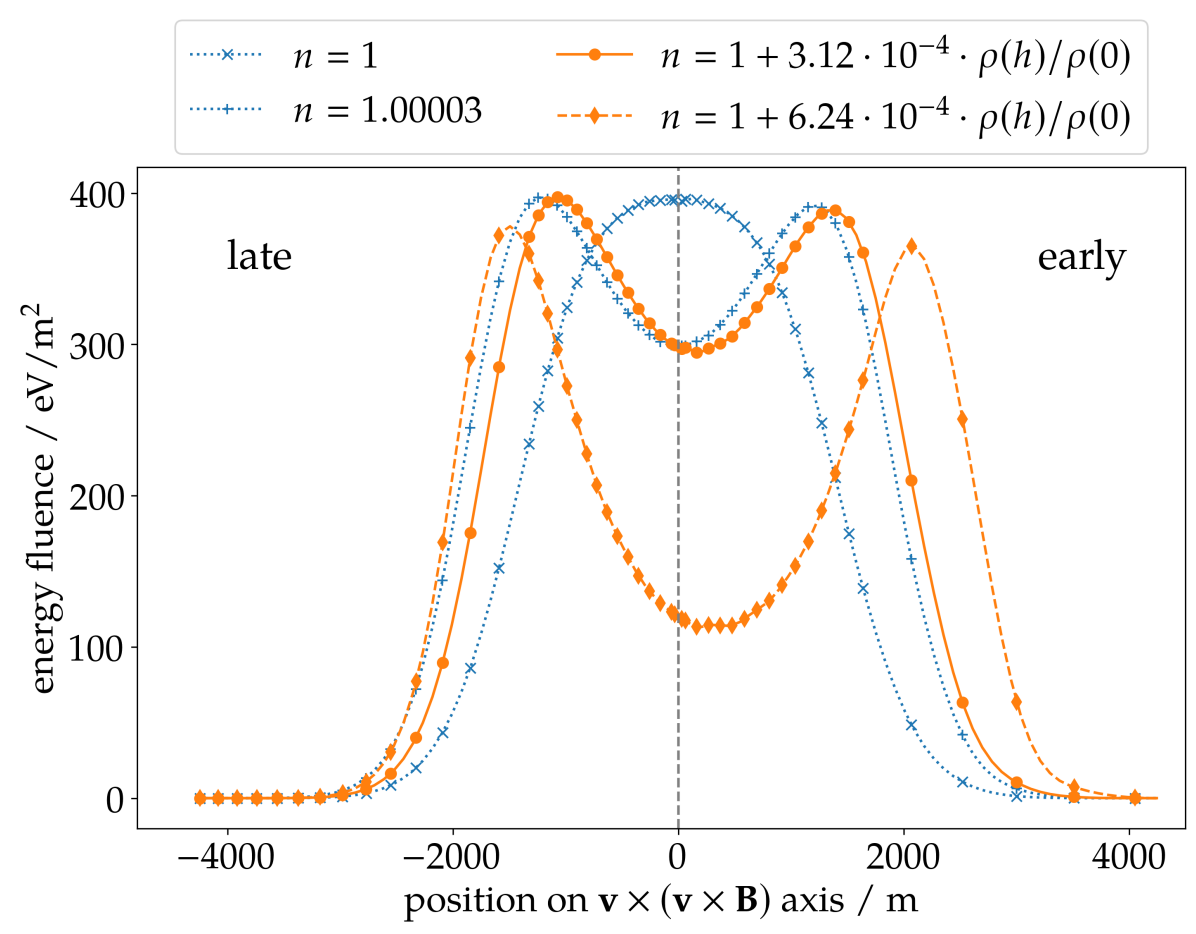

Figure 1: Lateral distribution of the radio emission along the positive and negative $\mathbf{v} \times(\mathbf{v} \times \mathbf{B})$ axis with respect to the MC impact point. Simulations with a refractive index changing with height are shown in orange, blue denotes a constant value of the refractive index. Figure updated from [5]. 
As there is no established model for the signal distribution of inclined air showers we use a purely geometrical approach that utilizes the Cherenkov-like compression of the radio signal [1]. On a ring around the shower axis, the Cherenkov ring, the signal strength is enhanced as a large fraction of the radio emission which is released during the complete shower evolution arrives simultaneously. To determine the displacement of the radio emission, we estimate its center by fitting a ring to the positions of the maximal energy fluence and define the center of this ring as our radio core. The found displacement, i.e. the distance between the fitted radio core and the MC impact point, is compared to predictions of a model that describes the propagation of the radio emission through a refractive atmosphere based on Snell's law.

In this proceeding, we only refer to the frequency band of the radio emission from $30 \mathrm{MHz}$ to $80 \mathrm{MHz}$ as used by most current-generation large-scale radio detector arrays. The simulation settings, i.e. the observation level of $1400 \mathrm{~m}$ above sea level, the Malargüe October atmospheric model and the refractivity at sea level $n_{0}-1=3.12 \times 10^{-4}$, match the ambient conditions of the Pierre Auger Observatory [6].

\section{Fitting the Cherenkov ring}

The radio emission is simulated with CORSIKA [7] (pre-release version of the package V7.7000) and its CoREAS extension [3] for observers on a star-shaped grid, i.e. the observers are located along the $\mathbf{v} \times \mathbf{B}$ and $\mathbf{v} \times(\mathbf{v} \times \mathbf{B})$ axes and their bisections on concentric rings in the shower plane projected onto the ground. The spacing is denser close to the shower axis to sample the energy fluence distribution precisely and sparser outside, cf. Fig. 1 and Fig. 2. In the following we will refer to the pulses with the same polar angle with respect to the MC impact point in the shower plane as "arms" of the star-shaped grid.

The radius of the Cherenkov ring depends among other aspects on the geometrical distance to the source region. Since the geomagnetic and charge-excess emission originate from slightly different regions in the atmosphere [8] it is expected that both emission contributions exhibit an independent Cherenkov ring. Thus, we will describe the radio-emission footprint in terms of the geomagnetic energy fluence $f_{\text {geo }}$ and charge-excess energy fluence $f_{\text {ce }}$ separately. Both can be calculated from the energy fluence in the $\mathbf{v} \times \mathbf{B}$ and $\mathbf{v} \times(\mathbf{v} \times \mathbf{B})$ polarisation, $f_{\mathbf{v} \times \mathbf{B}}$ and $f_{\mathbf{v} \times(\mathbf{v} \times \mathbf{B})}$ respectively, via (derived from [9])

$$
f_{\mathrm{geo}}=\left(\sqrt{f_{\mathbf{v} \times \mathbf{B}}}-\frac{\cos \Phi}{|\sin \Phi|} \cdot \sqrt{f_{\mathbf{v} \times(\mathbf{v} \times \mathbf{B})}}\right)^{2} \quad \text { and } \quad f_{\mathrm{ce}}=\frac{1}{\sin ^{2} \Phi} \cdot f_{\mathbf{v} \times(\mathbf{v} \times \mathbf{B})}
$$

where $\Phi$ denotes the polar angle of the pulse position in the shower plane with respect to the positive $\mathbf{v} \times \mathbf{B}$ axis counting counterclockwise.

An example shower with a small geomagnetic angle $\alpha$ (angle between the magnetic field axis and shower axis) is shown in Fig. 2. For showers with such a weak geomagnetic contribution, $\sin \alpha \approx$ 0.19 , the interference between geomagnetic and charge-excess emission impacts the position of the maximal fluence for the total radio emission and can even completely suppress the Cherenkov ring in the negative $\mathbf{v} \times \mathbf{B}$ half-plane. In contrast, the geomagnetic and charge-excess energy fluences individually exhibit a clear maximum. Thus, we fit the Cherenkov ring only to the dominating geomagnetic contribution of the radio emission. 


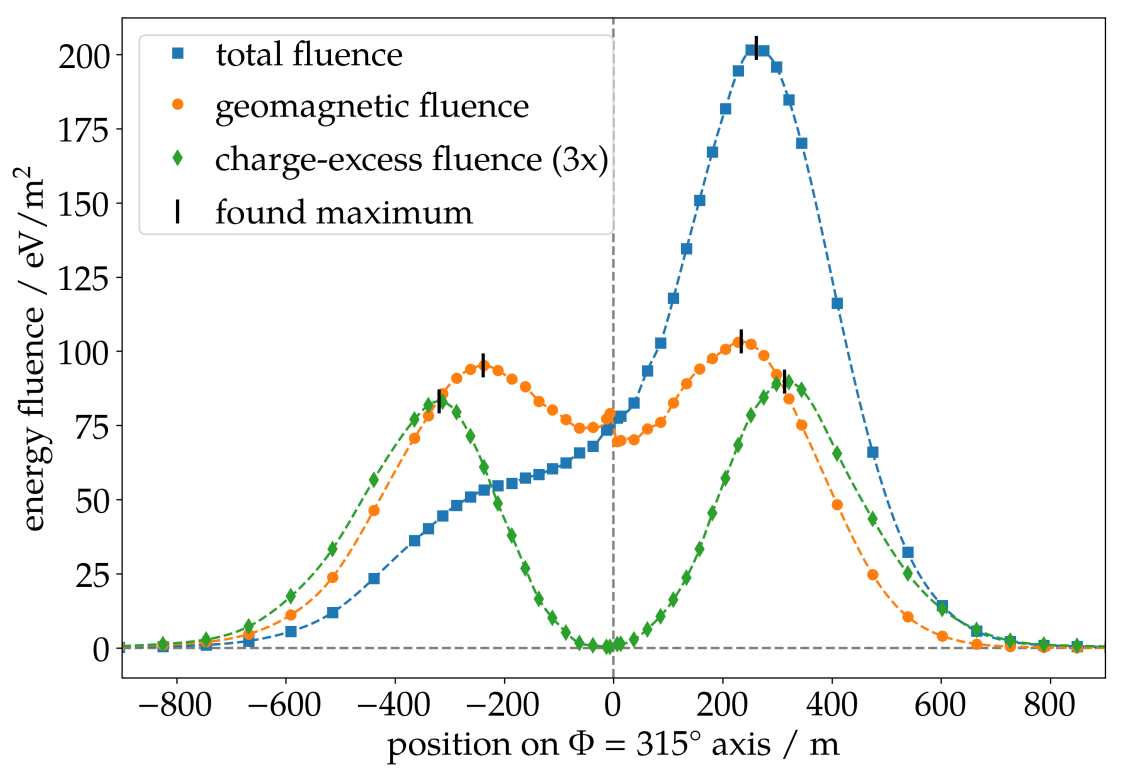

Figure 2: Comparison of the geomagnetic, charge-excess and total energy fluence (cf. eq. (1)) for a shower coming from North with a zenith angle of $65^{\circ}$. This corresponds to a geomagnetic angle with $\sin \alpha \approx 0.19$. Observers are shown on an axis with $\Phi=315^{\circ}$, negative values correspond to the $\Phi=135^{\circ}$ axis. The found maxima are marked by black vertical lines on the LDF. For the geomagnetic energy fluence, a non-physical behavior can be seen close to the axis. This is an artifact of using the MC impact point as the radio symmetry center in the calculation.

However, the calculation of the geomagnetic energy fluence depends on the polar angle of the station in the shower plane and therefore also on the radio core. We will therefore use an iterative fit that first calculates the geomagnetic energy fluence using the MC impact point as an estimation for the radio core, then fits the Cherenkov ring and estimates a new radio core. The Cherenkov ring is described by the position along each arm of the star-shaped grid, for which the energy fluence is maximal. These positions are found using a cubic spline interpolation. For the minimization process we employ a least-squares method with equal weights for each ring position. The $\mathbf{v} \times \mathbf{B}$ axis is excluded from the fit as the calculation of $f_{\text {geo }}$ and $f_{\text {ce }}$ following eq. (1) becomes nonphysical for small values of $\sin \Phi$.

An example fit to the geomagnetic emission for an event with a zenith angle of $85^{\circ}$ coming from North-West is shown in Fig. 3. The displacement between the radio core and MC impact point is estimated as $(125 \pm 21) \mathrm{m}$ in the shower plane which corresponds to a displacement of $(1428 \pm 240) \mathrm{m}$ on the ground due to the high inclination. Using the Monte-Carlo impact point the maximal difference between the Cherenkov radii, found on the individual arms, amounts to $268 \mathrm{~m}$. This difference reduces to $40 \mathrm{~m}$ with the radio core.

\section{Displacement of the radio-emission footprint}

The displacement is analyzed using a set of 4308 inclined air showers. The simulations contain proton and iron primaries with energies $18.4 \leq \log _{10}(E / \mathrm{eV}) \leq 20.2$ in $\log _{10}(E / \mathrm{eV})=0.2$ steps, binned zenith angles from $65^{\circ}$ to $85^{\circ}$ with a step size of $2.5^{\circ}$, and 8 equidistantly spaced azimuth 

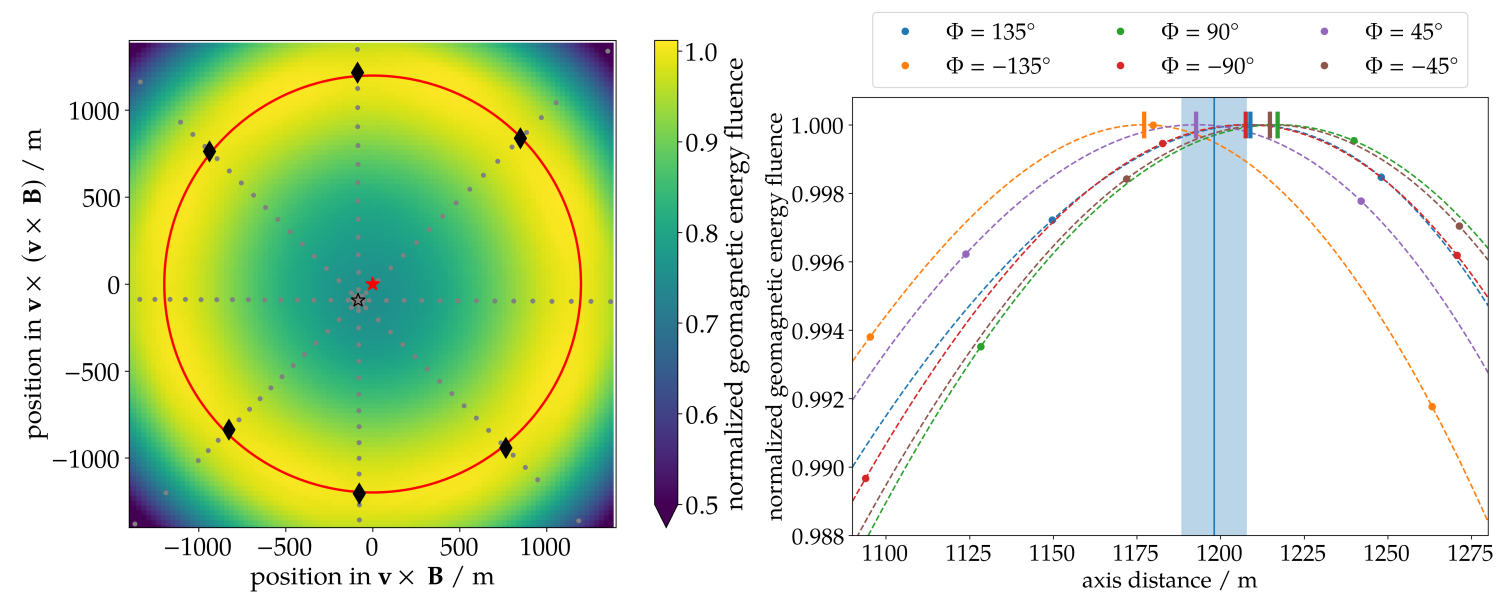

Figure 3: Result of the iterative fit procedure. The geomagnetic energy fluence is normalized to the maximum along each arm. For the fit, only the position of the Cherenkov ring along the arm, and not its signal strength, is used. 2D visualization of the fitted Cherenkov ring (left). For illustration purposes the background constitutes the cubic interpolation of the geomagnetic energy fluence. The fitted Cherenkov ring and its center are shown in red. The black star marks the position of the MC impact point, grey dots show the positions of the simulated pulses. The positions of maximal geomagnetic energy fluence found for each arm of the star-shaped grid are denoted by the black diamonds. 1D lateral distribution of the geomagnetic energy fluence (right). Colored points denote the calculated geomagnetic energy fluence for the simulated pulses. Their interpolation is shown by the dashed lines for each arm, the position of the maximum geomagnetic energy fluence is marked by the colored vertical line. The blue line and box denote the fitted radius of the Cherenkov ring and its uncertainty. The axis distances displayed on the $x$-axis are calculated using the fitted radio symmetry center.

angles $\phi$, i.e. coming from geomagnetic East $\left(\phi=0^{\circ}\right)$, North-East $\left(\phi=45^{\circ}\right)$, North $\left(\phi=90^{\circ}\right)$, etc. For showers with a small geomagnetic angle it is challenging to disentangle the contributions of the geomagnetic and the charge-excess emission. We will therefore only analyze air showers with a large geomagnetic angle, i.e. $\sin \alpha \geq 0.25$. This yields 4185 events with an accurate fit of the Cherenkov ring.

We interpret our results as function of the geometric distance $d_{\max }$ from the MC impact point to $X_{\max }$. In the first order $d_{\max }$ scales with the zenith angle, and only in second order with $X_{\max }$. Given $X_{\max }$ as input, it can only be calculated numerically for inclined air showers as the atmospheric curvature needs to be taken into account by solving

$$
X_{\text {ground }}-X_{\max }=\int_{0}^{d_{\max }} \rho(\ell) \mathrm{d} \ell .
$$

The atmospheric slant depth measured along the shower axis of the ground plane is denoted by $X_{\text {ground }}, \rho(\ell)$ denotes the atmospheric density at the distance $\ell$ along the MC shower axis in the direction of $X_{\max }$.

The observed displacement between the MC impact point and the radio core is shown in Fig. 4. The displacement increases with the inclination of the air shower up to a ground distance of more than $1500 \mathrm{~m}$ for $\theta=85^{\circ}$. This corresponds to an displacement in the shower plane of $15 \%$ of the fitted Cherenkov radius. A pronounced scatter is found that depends on the azimuth angle. The 

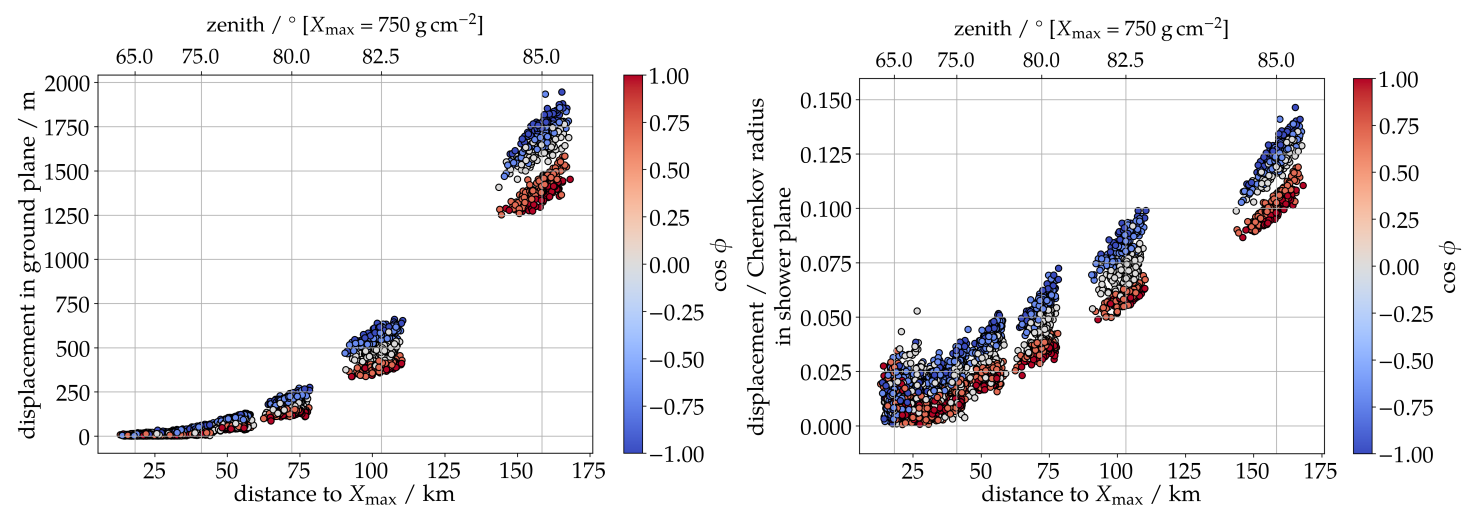

Figure 4: Displacement of the radio core with respect to the $\mathrm{MC}$ impact point in the ground plane (left) and in the shower plane normalized to the fitted radius of the Cherenkov ring (right) as function of distance to $X_{\max }$. The color-coded cosine of the azimuth arrival direction illustrates an East-West asymmetry.

displacement is strongest for air showers coming from West $(\cos \phi=-1)$ and weakest for East $(\cos \phi=1)$.

This asymmetry is also seen in the position of the fitted radio core with respect to the MC impact point in the coordinate origin, cf. Fig. 5. The radio core is always displaced towards the incoming direction of the air shower, i.e. towards the shower maximum. The radio core of an air shower coming from the West is stronger displaced from the MC impact point than an air shower coming from the East with a similar inclination. In addition, a small rotation of the pattern can be seen. The exact origin of these asymmetries needs further investigation, they cannot be caused by atmospheric properties as the atmosphere in CoREAS simulations is rotationally symmetric.

\section{Interpretation of the displacement as due to refraction}

We will now show that the observed displacement is in agreement with refraction of radio waves in a refractive atmosphere as described by Snell's law. Therefore, we develop a model simulating the propagation of a single electromagnetic wave through the Earth's atmosphere. We use a curved atmosphere and assume discrete changes of the refractive index at the edges of imaginary layers throughout the atmosphere with a thickness of $1 \mathrm{~m}$ to ensure high accuracy of the calculation. The propagation within a layer with an upper edge height $h_{i}$ is described by a straight uniform expansion with the phase velocity $c_{n}=c_{0} / n\left(h_{i}\right)$ given the refractive index as function of the height above sea level $n(h)$. The change of direction between two layers with refractive indices, $n_{1}$ and $n_{2}$, respectively, is described in terms of the incidence angle, $\vartheta$, from $\vartheta_{1}$ to $\vartheta_{2}$ following Snell's law

$$
\frac{\sin \vartheta_{2}}{\sin \vartheta_{1}}=\frac{n_{1}}{n_{2}}
$$

The initial direction of the electromagnetic wave is aligned with the MC axis of the air shower. The displacement can be inferred from the distance of the intersection between the bent trajectory and the ground plane and the intersection between the MC axis and the ground plane. By construction, the model always predicts a displacement towards the incoming direction of the air shower. 


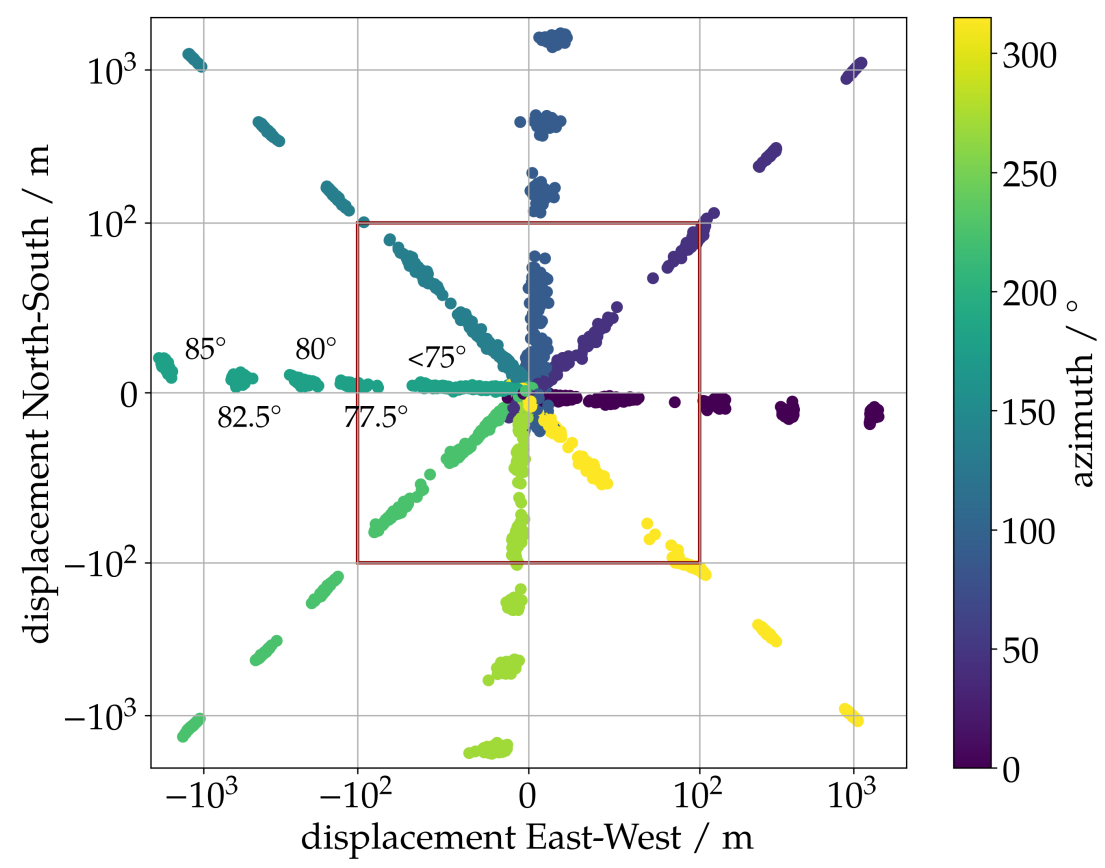

Figure 5: Displacement of the radio core in the ground plane relative to the MC impact point in the coordinate origin. Distinguishable zenith angle bins are annotated in the plots The radio symmetry center is always displaced into the incoming direction of the showers. The displacement is shown with a linear scale within the first $100 \mathrm{~m}$ (red square) and with a logarithmic scale afterwards.

The predicted displacement in the ground plane is shown in Fig. 6 for inclinations between $65^{\circ}$ and $85^{\circ}$. The orange line symbolizes the displacement for a source at a fixed slant depth of $750 \mathrm{~g} / \mathrm{cm}^{2}$ which corresponds to the average $X_{\max }$ value in our data sample. The orange squares denote the displacement for different slant depths between $620 \mathrm{~g} / \mathrm{cm}^{2}$ and $1000 \mathrm{~g} / \mathrm{cm}^{2}$ (typical range in our data sample) along the MC axis for five different zenith angles $\left(\theta=65^{\circ}, 75^{\circ}, 80^{\circ}, 82.5^{\circ}\right.$, $85^{\circ}$ ). The displacement is reasonably described by our model in terms of the overall magnitude as well as the slope as function of the source's slant depth. The residuals in the bottom frame show no strong correlation with depth of shower maximum and increase up to $\sim 250 \mathrm{~m}$ for the most inclined showers.

\section{Conclusions}

For inclined air showers the radio core is displaced up to $1500 \mathrm{~m}$ from the MC impact point due to refraction of the radio emission in the atmosphere. The found displacement in simulations agrees with the calculation of a model describing the propagation of the radio emission through the atmosphere as a continuous reflection following Snell's law in a curved atmosphere. These findings have several implications towards the observation of cosmic rays with the radio detection technique. Assuming the MC impact point as symmetry center of the radio-emission footprint will lead to a mismodelling of the signal distribution which is relevant e.g. for the development of new reconstruction algorithms. The displacement has to be taken into account for a hybrid detection of an inclined air shower with different detection techniques. 


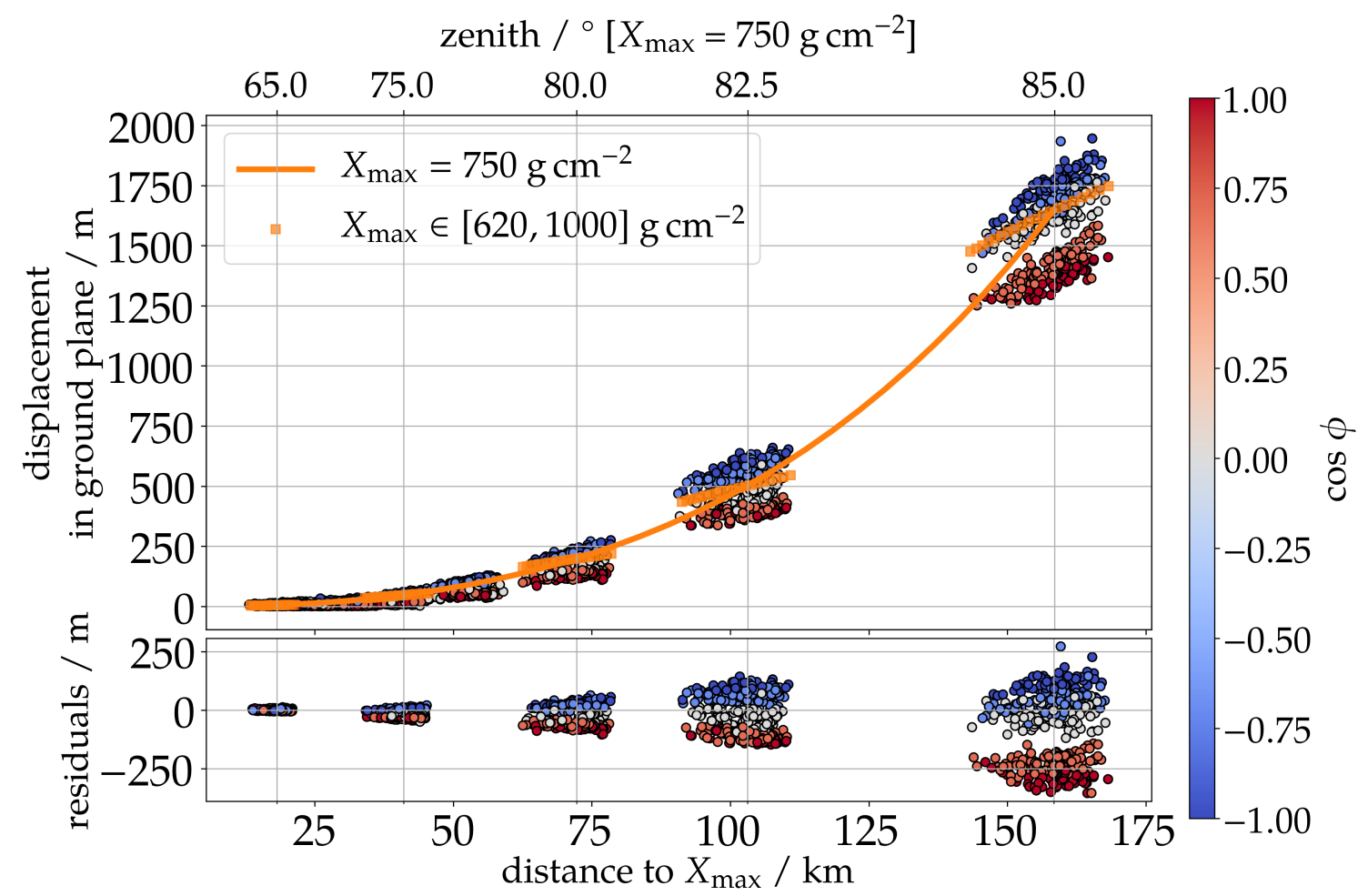

Figure 6: Comparison between model-predicted (orange) and CoREAS-derived (colored circles, same as Fig. 4 (left)) displacement of the radio core in the ground plane. The residuals are shown in the bottom frame.

More information on the displacement can be found in [4]. There, the dependence of the displacement is also studied for the radio emission in the $50 \mathrm{MHz}$ to $200 \mathrm{MHz}$ band as proposed for many next generation radio experiments such as GRAND [10]. We find a similar displacement of the radio emission and a $\sim 5 \%$ increase of the average Cherenkov radius for the higher frequency band. The contributions of the geomagnetic and charge-excess emissions are studied individually, the estimated radius of the Cherenkov ring is systematically larger for the charge-excess contribution than for the geomagnetic emission. The model used for the prediction of the displacement is described in depth and the treatment of the refractivity in CoREAS is validated.

\section{References}

[1] T. Huege. Phys. Rept. 620 (2016) 1-52

[2] T. Huege, L. Brenk, and F. Schlüter. EPJ Web Conf, 216 (2019) 03009

[3] T. Huege, M. Ludwig, and C. W. James, AIPConf. Proc., 1535 (2013) 128

[4] F. Schlüter et al. EPJC 80 (2020) 643

[5] M. Gottowik on behalf of the Pierre Auger Collaboration, PoS(ICRC2019)274

[6] The Pierre Auger Collaboration. Nucl. Instrum. Meth. A, 798 (2015) 172 - 213

[7] D. Heck et al. FZKA Report 6019, Forschungszentrum Karlsruhe, 1998

[8] C. Glaser et al. JCAP, 1609 (2016) 024

[9] C. Glaser et al. Astropart. Phys., 104 (2018) 64-77

[10] J. Álvarez Muñiz et al. Sci. China Phys. Mech. Astron. 63 (2020) 219501 